\title{
PREFACE
}

\section{Background to the INTERMAP Study of nutrients and blood pressure}

\author{
DG Beevers ${ }^{1}$ and J Stamler ${ }^{2}$ \\ ${ }^{1}$ University of Birmingham, UK; ${ }^{2}$ Department of Preventive Medicine, Feinberg School of Medicine, \\ Northwestern University, Chicago, IL, USA
}

Journal of Human Hypertension (2003) 17, 589-590. doi:10.1038/sj.jhh.1001609

Countless epidemiological surveys have shown that there are striking interindividual and interpopulation differences in blood pressure. ${ }^{1-3}$ In most - but not all-populations, blood pressure generally rises (more or less) with age from youth into older age. The exceptions are isolated preliterate groups in remote locations, where average systolic and diastolic blood pressures are optimal at all adult ages, manifesting little or no upward slope with age - and where lifestyles differ markedly compared to those of other populations worldwide. ${ }^{4,5}$ Data from migration studies - for example, the Luo Migrant Study in Kenya and the Ye Migrant Study in China - strongly indicate that changes in lifestyle and nutrition explain increases in blood pressure and vascular disease following migration and adoption of diets broadly similar to those of host populations. ${ }^{6,7}$ Furthermore, an inverse relation between socioeconomic status (SES) and blood pressure has also been recorded repeatedly in many population studies of specific ethnic groups, for example, African Americans and non-Hispanic white Americans. ${ }^{8-10}$ All these findings support the conclusion that such group differences in blood pressure have little or no basis in population genetics (whatever the role of interindividual genetic differences in accounting for interindividual BP differences). Thus, lifestyle and dietary factors are likely to be important.

Investigation into the nature of the nutritional and other lifestyle factors that might explain interindividual and interpopulation differences in blood pressure is difficult to conduct. Particularly when the objective is to study large samples drawn from diverse populations, surveys inevitably have to be conducted at several sites, usually by multiple investigators. This being so, differences in research methods, population selection, and a whole galaxy of potential confounding variables may open to question much well-meaning research. These problems to a large extent led to the doubts expressed years ago by Sir George Pickering and others on the validity of the salt hypothesis.
It was in the light of these concerns that the INTERSALT project was created. In this study, $10000+$ men and women aged 20-59 years from 52 discrete population samples in 32 countries were investigated in order to assess in particular the role of salt intake in accounting for differences - interindividual $(N=10000+)$ and interpopulation $(N=52)$ - in blood pressure.$^{5,10-14}$ Rigorous standardization of blood pressure measurement techniques as well as ascertainment of salt intake together with the possible confounders of alcohol and potassium intake and body mass index (BMI) were mandatory. All biochemical tests were conducted in a single laboratory. This study was able to demonstrate significant independent relationships between salt and blood pressure across its $10000+$ individual participants. It also showed-for the first time - that higher salt and alcohol intake, greater BMI, and lower potassium intake of less educated individuals accounted significantly for their higher blood pressures. ${ }^{10}$ In the cross-population (ecologic) analyses $(N=52)$, there were significant relationships between population sample average salt intake and population sample blood pressure variables, including - notably - a strong relation of salt to upward slope of blood pressure from young adulthood into middle age. ${ }^{11-13}$ The success of the INTERSALT project did silence many (but not all) of the doubters. We were delighted [in the Journal of Human Hypertension] to be able to publish several INTERSALT papers as well as detailed "raw" data in 64 tables providing investigators elsewhere with the opportunity to examine and analyse our data. ${ }^{12}$

Perhaps another important achievement of the INTERSALT project was the creation of an international cadre of well-trained and motivated investigators, keen to continue epidemiological research using the same high standards. It also proved that it is possible to conduct research of this nature, including individuals from population samples of unacculturated and remote societies, economically developing countries, and highly industrialized nations. 
The INTERSALT project provided answers on salt, potassium, alcohol, and body mass, elucidating their role in interindividual and interpopulation differences in blood pressure, including in relation to socioeconomic status. However, multiple other dietary factors of potential relevance were not assessed. In part the phase-2 INTERSALT observation of an inverse association between 24-h urinary nitrogen excretion (a marker of protein intake) of individuals and their blood pressures prompted the INTERSALT investigators to feel that further research was in order. ${ }^{14}$

Thus out of INTERSALT, INTERMAP was born. The focus was to be only on individuals - the role of multiple dietary factors in accounting for interindividual differences in blood pressure. This time it was decided to study diverse population samples in only four nations (China, Japan, UK, USA), but to do so in great detail. Comprehensive high-quality databases on the nutrient composition of foods had to be developed, based on already extant national tables - a huge task not feasible for many of the original INTERSALT populations. With INTERMAP it was decided to conduct an in-depth nutritional study involving four 24-h dietary recalls as well as two 24-h urine collections. Again, standardization of techniques had to be rigorous. All potential investigators had to be trained or retrained in blood pressure measurement as well as the accurate assessment of the intake of all foods, beverages, and dietary supplements, their nature and their quantity. Quality control of data gathering had to be assiduous, with site visits, double checks, and regular assessments of accuracy of survey techniques. The quest for accuracy in epidemiological techniques may seem at times trivial and 'pettyfogging' to clinicians, but failure to achieve standardization of survey techniques can confound a whole project and waste everyone's time.

Extensive data are now in hand and multiple analyses have been made. Once again, we are proud at the Journal of Human Hypertension to be able to publish comprehensive tabulations of the descriptive data, together with six of the papers. We hope that investigators elsewhere will find our detailed data useful for their own research.

\section{References}

1 Stamler J, Stamler R, Pullman TN. The Epidemiology of Hypertension. Grune and Stratton: New York, 1967.
2 Paul O (ed). Epidemiology and Control of Hypertension. Symposia Specialists: Miami, 1975.

3 Stamler J. Blood pressure levels in and across populations. NHLBI-sponsored Satellite Symposium to the International Society of Hypertension Meeting, Bethesda, MD, August 25, 2000; published by NHLBI on the Internet (http://www.nhlbi.nih.gov/meetings/ish/ stamler.htm).

4 Shaper AG. Communities without hypertension. In: Shaper AG, Hutt MSR, Fejfar Z (eds). Cardiovascular Disease in the Tropics. British Medical Association: London, 1974, pp 77-83.

5 Mancilha-Carvalho JJ et al. Blood pressure in four remote populations in the INTERSALT Study. Hypertension 1989; 14: 238-246.

6 Poulter $\mathrm{N}$ et al. Blood pressure and its correlates in an African tribe in urban and rural environments. J Epidemiol Community Health 1984; 38: 181185.

$7 \mathrm{He}$ J et al. Effect of migration on blood pressure: the Yi People Study. Epidemiology 1991; 2: 88-97.

8 Dyer AR, Stamler J, Shekelle RB, Schoenberger J. The relationship of education to blood pressure: findings on 40,000 employed Chicagoans. Circulation 1976; 54: 987-992.

9 Hypertension Detection and Follow-up Program Cooperative Group. Educational level and 5-year allcause mortality in the Hypertension Detection and Follow-up Program. Hypertension 1987; 9: 641646.

10 Stamler $\mathrm{R}$ et al, on behalf of the INTERSALT Cooperative Research Group. Higher blood pressure in adults with less education: some explanatory factors. Findings of the INTERSALT Study. Hypertension 1992; 19: 237-241.

11 INTERSALT Cooperative Research Group. INTERSALT: an international study of electrolyte excretion and blood pressure. Results for 24-hour urinary sodium and potassium excretion. BMJ 1988; 297: 319-328.

12 INTERSALT Cooperative Research Group (Elliott P, Guest ed). The INTERSALT Study - an international cooperative study of electrolyte excretion and blood pressure: further results. J Hum Hypertens 1989; 3: 279-407.

13 Stamler J. The INTERSALT Study: background, methods, findings, and implications. Am J Clin Nutr 1997; 65 (2 Suppl): 626S-642S.

14 Stamler J et al, for the INTERSALT Cooperative Research Group. Inverse relation of dietary protein markers with blood pressure. Findings for 10,020 men and women in the INTERSALT Study. Circulation 1996; 94: 1629-1634. 